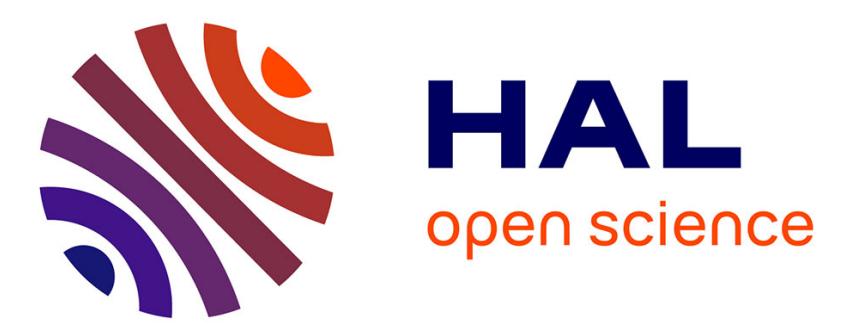

\title{
Influence de l'indice de masse corporelle sur les troubles urinaires de la sclérose en plaques
}

G. Miget, C. Hentzen, N. Turmel, C. Chesnel, A. Charlanes, F. Le Breton, G. Amarenco

\section{- To cite this version:}

G. Miget, C. Hentzen, N. Turmel, C. Chesnel, A. Charlanes, et al.. Influence de l'indice de masse corporelle sur les troubles urinaires de la sclérose en plaques. Progrès en Urologie, 2019, 29, pp.366 370. 10.1016/j.purol.2019.04.004 . hal-03487177

\section{HAL Id: hal-03487177 \\ https://hal.science/hal-03487177}

Submitted on 20 Dec 2021

HAL is a multi-disciplinary open access archive for the deposit and dissemination of scientific research documents, whether they are published or not. The documents may come from teaching and research institutions in France or abroad, or from public or private research centers.
L'archive ouverte pluridisciplinaire HAL, est destinée au dépôt et à la diffusion de documents scientifiques de niveau recherche, publiés ou non, émanant des établissements d'enseignement et de recherche français ou étrangers, des laboratoires publics ou privés.

\section{(ㄷ)(1) $\$$}

Distributed under a Creative Commons Attribution - NonCommerciall 4.0 International 
Influence de l'indice de masse corporelle sur les troubles urinaires de la sclérose en plaques.

Gabriel Miget ${ }^{1,2}$, Claire Hentzen ${ }^{1,2}$, Nicolas Turmel ${ }^{1,2}$, Camille Chesnel $^{1,2}$, Audrey Charlanes ${ }^{1,2}$, Frédérique Le Breton ${ }^{1,2}$, Gérard Amarenco ${ }^{1,2}$

${ }^{1}$ Sorbonne Université, GRC 001, GREEN Groupe de Recherche Clinique en Neuro-Urologie, AP-HP, Hôpital Tenon, F-75020 Paris, France

${ }^{2}$ Service de Neuro-Urologie et d'Explorations Périnéales, AP-HP, Hôpital Tenon, 75020 Paris, France

Auteur correspondant :

MIGET Gabriel

gabrielmiget@gmail.com

Téléphone : 0156017500

Fax : 0156017481 


\section{Introduction}

Les troubles vésico-sphinctériens (TVS) sont une problématique fréquente de la sclérose en plaques (SEP) puisque 10\% des sujets présentent des TVS dès l'apparition des premiers symptômes et $100 \%$ des patients à 10 ans d'évolution de la maladie (1). Leur typologie est bien connue avec une fréquence de l'hyperactivité vésicale (HAV, 26\% à 99\%) et de la dysurie $(6 \%$ à $49 \%)(2,3)$. Pour autant, l'origine des TVS n'est pas forcément univoque car nombre de comorbidités peuvent-être associées et particulièrement l'obésité qui représente un facteur de risque d'incontinence urinaire d'effort (IUE) (4) et qui favorise également I'HAV (5). Devant la prévalence élevée constatée du surpoids et de l'obésité dans la population SEP (6), nous avons souhaité évaluer l'influence de l'indice de masse corporelle (IMC) sur les troubles urinaires de la SEP au moyen du score USP (7).

\section{Matériels et Méthodes}

Nous avons réalisé une étude rétrospective monocentrique, chez 260 sujets atteints d'une SEP, ayant consulté pour troubles urinaires dans un service spécialisé de neuro-urologie. L'objectif principal de cette étude était d'évaluer l'influence de l'IMC sur les différentes dimensions du score USP (HAV, IUE et dysurie), questionnaire validé dans la population neurologique (7). Différentes comparaisons ont été effectuées, en fonction du sexe et de la classe d'IMC selon la classification de l'OMS : IMC $\left(\mathrm{kg} / \mathrm{m}^{2}\right)<18,5=$ insuffisance pondérale, $18,5 \leq \mathrm{IMC}<25$ = poids normal, $25 \leq \mathrm{IMC}<30$ surpoids, IMC $\geq 30$ = obésité.

Nous avons utilisé des tests de comparaisons de moyennes par test de Mann Whitney, compte tenu d'une positivité du test de Shapiro-Wilk concernant la distribution de l'IMC, ne permettant donc pas d'utiliser le test de Student. Les tests de corrélation entre IMC et scores USP ont été effectuée par calcul du coefficient de corrélation de Pearson. Les 
analyses statistiques ont été effectuées de façon bilatérale avec un seuil de significativité alpha de 0,05. L'ensemble des tests statistiques ont été effectués sur RStudio : RStudio Team (2015). RStudio : Integrated Developpement Environnement for R. R Studio, Inc., Boston, MA URL http://www.rstudio.com/.

\section{Résultats}

Cent quatre-vingt-six femmes (71\%) et 74 hommes (28\%) soit 260 sujets ont été inclus. L'âge moyen était de 50 ans ( $S D=12,6)$, le score EDSS moyen était de 4,86 (SD=1,81) et l'IMC moyen de $24,48 \mathrm{~kg} / \mathrm{m}^{2}(\mathrm{SD}=4,6)$. Vingt sujets (7\%) présentaient une insuffisance pondérale, 126 sujets (48\%) avaient un poids normal, 84 (32\%) présentaient un surpoids et 24 (9\%) étaient obèses.

Les scores moyens par chaque classe d'IMC de la population globale sont décrits en figure 1. Les sujets obèses avaient un score HAV moyen de $10,27(S D=4,50)$ statistiquement plus élevé que les sujets présentant une insuffisance pondérale, HAV moyen = 5,75 $(S D=3,43), p=$ 0,001 , ainsi que les sujets présentant un poids normal, HAV moyen $=7,40(S D=4,63) p=$ 0,0036. Ce score était également inférieur chez les sujets en surpoids, HAV moyen $=7,19$ $(S D=4,44) p=0,0028$

La même observation a été effectuée concernant le score IUE chez les sujets obèses. Ils présentaient un score IUE moyen de $3,80(S D=3,23)$, plus élevé que les sujets ayant une insuffisance pondérale, IUE moyen $=1,45(S D=2,01), p=0,0062$, ainsi que les sujets présentant un poids normal, IUE moyen $=1,48(S D=2,27), p=0.00004$. Les sujets en surpoids avaient également un score inférieur, IUE moyen =2,12 (SD=2,69), $p=0,0058$. 
Concernant le score dysurie, celui-ci était statistiquement plus élevé chez les sujets avec un poids normal avec un score moyen de 4,08 $(S D=3,17)$. Les sujets présentant un surpoids avaient un score moyen de 2,64 ( $S D=2,61), p=0,001$ et les sujets obèses un de score de 2,33 $(S D=2,37), p=0,006$.

Les mêmes comparaisons ont été effectuées en fonction du sexe. La population féminine était composée de 20 (10\%) sujets présentant une insuffisance pondérale, 93 (50\%) avaient un poids normal, 43 (23\%) étaient en surpoids et 30 (16\%) étaient obèses. Les scores moyens sont décrits en figure 2. Chez la femme obèse, le score HAV moyen était plus élevé que les femmes ayant un poids normal : HAV moyen $=10,27(S D=4,5)$ vs $7,96(S D=4,58) p=$ 0,024 . On retrouvait également une différence entre les sujets présentant une insuffisance pondérale et les sujets en surpoids: $\operatorname{HAV}$ moyen $=5,75(\mathrm{SD}=3,43)$ vs $8,88(\mathrm{SD}=3,82) \mathrm{p}=$ 0,006 .

Concernant le sous score IUE, les femmes présentant un IMC dans la norme avaient un score moyen de 1,69 $(S D=2,38)$, significativement inférieur à celui du sous-groupe surpoids, IUE moyen $=3,19(S D=2,91), p=0,002$ et également inférieur au groupe obèses, IUE moyen $=$ 3,80 ( $S D=3,23), p=0,0005$. La même observation était effectuée concernant le groupe insuffisance pondérale, score IUE $=1,45(S D=2,01)$, en comparaison aux groupes surpoids, $p$ $=0,016$ et obèses, $p=0,006$.

En revanche, comme dans la population globale, le score dysurie, était statistiquement plus élevé chez les sujets ayant un poids normal avec un score dysurie moyen $=4,09(S D=3,33)$ que chez les sujets présentant un surpoids, USP dysurie $=1,91(S D=2,03), p=0,0003$ ainsi que chez les sujets obèses, USP dysurie $=2,33(S D=2,37), p=0,013$. La même observation était effectuée concernant le groupe insuffisance pondérale, score dysurie $=3,55(S D=2,51)$, en comparaison aux groupes surpoids, $p=0,0065$ et obèses, $p=0,025$. 
Par ailleurs, il existait une corrélation directe entre IMC et score HAV, $p=4,1 \mathrm{e}^{-5}, \rho=0,29, \mathrm{IC}$ $95 \%[0,15-0,42]$ ainsi qu'entre IMC et le score IUE, $p=1,6 \mathrm{e}^{-7}, \rho=0,37, \mathrm{IC} 95 \%[0,24-0,49]$. A contrario, on notait une corrélation indirecte entre le score dysurie et l'IMC, $p=7 e^{-4}, \rho=-$ 0,24, IC $95 \%[-0,38-0,10]$.

Les mêmes analyses effectuées chez l'homme ne montraient pas de différence significative.

\section{Discussion}

Cette étude met en évidence un lien entre I'IMC et les troubles urinaires de la SEP et particulièrement chez la femme. En effet, plus l'IMC était élevé, plus le score HAV moyen était élevé, traduisant ainsi un syndrome clinique d'HAV plus sévère. La non significativité des résultats chez l'homme peut en partie être expliquée par l'absence de sujets obèses (classification de l'OMS) dans notre population masculine, avec aucun homme ayant un IMC supérieur à $30 \mathrm{~kg} / \mathrm{m}^{2}$.

Les résultats observés chez la femme, en particulier concernant le score HAV, soulignent que l'étiopathogénie des TVS n'est pas toujours univoque. Bien que la participation neurologique liée à l'atteinte inflammatoire démyélinisante du système nerveux central (SNC) soit évidente, certaines comorbidités telles que l'HTA (23\%), la dyslipidémie $(31,1 \%)$ et donc l'obésité $(22,5 \%)$ sont fréquemment observées chez la $\operatorname{SEP}(8,9)$, définissant un syndrome métabolique (30\%) lorsqu'elles sont associées $(10,11)$. L'impact du syndrome métabolique dans le déterminisme des TVS par des mécanismes multiples (inflammation chronique, dysfonction endothéliale, ischémie chronique, remaniements fibrotiques du détrusor) est aujourd'hui bien étudiés (11-13). Ces mécanismes sous-jacents pourraient être à l'origine, du moins en partie, du syndrome d'HAV ou encore favoriser l'hypertrophie bénigne de prostate chez I'homme $(11,12,14)$. 
Par ailleurs, l'atteinte du système nerveux autonome (SNA) constitue un dénominateur commun de diverses pathologies comme le diabète (15), l'HPB (16), l'obésité et le syndrome métabolique $(17,18)$. Cette atteinte du SNA, aussi observée au cours de la SEP, est à l'origine de TVS par déséquilibre de la balance sympathique/parasympathique. Le rôle de la ghréline, hormone peptidique, est aussi suspecté dans les mécanismes de l'HAV chez les sujets obèses (19).

Le score IUE était également plus élevé en cas d'IMC important. Ces résultats sont conformes aux données de la littérature $(4,20)$. Différents mécanismes liés à l'obésité sont évoqués pour expliquer ces observations, comme l'augmentation de la pression intraabdominale, qui, transmise à la vessie, faciliterait l'inversion du gradient de pression urétrovésical $(4,20,21)$. Des remaniements tissulaires sont également évoqués, notamment au niveau sphinctérien, avec, chez l'animal, la mise en évidence d'inclusions lipidiques intracellulaires concernant aussi bien les fibres musculaires striées que lisses du sphincter urétral $(21,22)$ et pouvant être à l'origine d'incompétence sphinctérienne voire de trouble de compliance urétrale. Ces phénomènes peuvent aussi être associées aux problématiques mécanistiques d'altération du plancher pelvien par l'hyperpression abdominale et I'hypermobilité urétrale par défaut de soutènement. Également, les atteintes microvasculaires liées à la dysfonction endothéliale et l'inflammation chronique secondaires à l'obésité et aux autres facteurs de risques cardio-vasculaires souvient associés, peuvent participer à un défaut de contrôle neurologique de la continence, et donc, favoriser l'IUE. Ces facteurs pourraient également, au moins en partie, expliquer la diminution du score moyen de dysurie lors l'augmentation de l'IMC dans notre population.

Notre étude comporte cependant certaines limites et en particulier son caractère rétrospectif qui n'a pas permis de prendre en compte d'autres variables potentiellement 
impliquées dans l'expression de la symptomatologie et ainsi influençant les scores (forme et durée d'évolution de la SEP, traitements à tropisme vésico-sphinctérien, présence d'une constipation/dyschésie associée dont on connait le retentissement sur les troubles vésicosphinctériens (hypersensibilité croisée), présence d'une HBP, de troubles statiques, parité et le mode d'accouchement...). Une étude prospective avec recueil de ces variables pourrait permettre de déterminer l'influence de chaque facteur dans l'expression des TVS chez le sujet SEP.

\section{Conclusion}

Cette étude permet de mettre en évidence le probable rôle de l'obésité dans la genèse des TVS de la SEP. Bien qu'il existe un rôle évident de l'atteinte neurologique démyélinisante dans la génération des TVS dans cette population, celle-ci ne doit pas occulter le dépistage, l'évaluation et la prise en charge des comorbidités associées dont l'obésité. Des évaluations et prises en charges diététiques proposées à titre systématique, pourraient participer à l'amélioration de ces TVS, des troubles ano-rectaux souvent associés et partant de la qualité de vie. Cela permettrait aussi d'optimiser la prise en charge du handicap, l'obésité étant reconnu comme facteur limitant sur le plan de l'autonomie (marche, soins corporels, accessibilité du périnée pour la réalisation des auto-sondages intermittents...). Une telle prise en charge ne devra pas méconnaitre le risque de majorer une sarcopénie souvent déjà présente (23-25), dans cette population ou le capital musculaire doit être préservé.

\section{Déclaration de liens d'intérêt :}

Les auteurs ne déclarent pas avoir de liens d'intérêts. 
Références :

1. Tornic, Jure \& Panicker Jalesh N. The Management of Lower Urinary Tract Dysfunction in Multiple Sclerosis. Current neurology and neuroscience reports, 2018; 18(8), 54.

2. Phé V, Chartier-Kastler E, Panicker JN. Management of neurogenic bladder in patients with multiple sclerosis. Nature Reviews Urology. 2016;13(5):275-88.

3. Amarenco G, de Sèze M, Ruffion A, Sheikh Ismael S. Clinical and urodynamic evaluations of urinary disorders in multiple sclerosis. Ann Phys Rehabil Med. 2014;57(5):277-87.

4. Deffieux, X., Hubeaux, K., \& Amarenco, G. Incontinence urinaire à l'effort de la femme: analyse des hypothèses physiopathologiques. J. Gynecol. Obstet. Biol. Reprod., 2008;37(2), 186-196.

5. Zacche MM, Giarenis I, Thiagamoorthy G, Robinson D, Cardozo L. Is there an association between aspects of the metabolic syndrome and overactive bladder? A prospective cohort study in women with lower urinary tract symptoms. Eur J Obstet Gynecol Reprod Biol. 2017;217:1-5

6. Ben-Zacharia AB. The Effects of Body Mass Index (BMI) on Multiple Sclerosis (MS) Progression. 2018;1(1):7. 
7. Haab F, Richard F, Amarenco G, Coloby P, Arnould B, Benmedjahed K, et al. Comprehensive Evaluation of Bladder and Urethral Dysfunction Symptoms: Development and Psychometric Validation of the Urinary Symptom Profile (USP) Questionnaire. Urology. 2008;71(4):646-56.

8. Sicras-Mainar, A., Ruíz-Beato, E., Navarro-Artieda, R., \& Maurino, J. Comorbidity and metabolic syndrome in patients with multiple sclerosis from Asturias and Catalonia, Spain. BMC neurology. 2017;17(1), 134.

9. Marrie RA, Cohen J, Stuve O, Trojano M, Sørensen PS, Reingold S, et al. A systematic review of the incidence and prevalence of comorbidity in multiple sclerosis: overview. Mult Scler. 2015;21(3):263-81.

10. Pinhas-Hamiel O, Livne M, Harari G, Achiron A. Prevalence of overweight, obesity and metabolic syndrome components in multiple sclerosis patients with significant disability. European Journal of Neurology. 2015;22(9):1275-9.

11. Sáenz Medina J, Carballido Rodríguez J. Review of the pathophysiological aspects involved in urological disease associated with metabolic syndrome. Actas Urol Esp. 2016;40(5):279-87.

12. Uzun H, Zorba OÜ. Metabolic Syndrome in Female Patients With Overactive Bladder. Urology. 2012;79(1):72-5. 
13. He Q, Babcook MA, Shukla S, Shankar E, Wang Z, Liu G, et al. Obesity-initiated metabolic syndrome promotes urinary voiding dysfunction in a mouse model. Prostate. 2016;76(11):964-76.

14. He Q, Wang Z, Liu G, Daneshgari F, MacLennan GT, Gupta S. Metabolic syndrome, inflammation and lower urinary tract symptoms: possible translational links. Prostate Cancer Prostatic Dis. 2016;19(1):7-13.

15. Sarma AV, Parsons JK, McVary K, Wei JT. Diabetes and benign prostatic hyperplasia/lower urinary tract symptoms--what do we know? J Urol. 2009;182(6 Suppl):S32-37.

16. MCVARY, KEVIN T., RADEMAKER, Alfred, LLOYD, Granville L., et al. Autonomic nervous system overactivity in men with lower urinary tract symptoms secondary to benign prostatic hyperplasia. The Journal of urology, 2005;174(4 Part 1): 1327-1333.

17. KUPELIAN, Varant, MCVARY, Kevin T., KAPLAN, Steven A., et al. Association of lower urinary tract symptoms and the metabolic syndrome: results from the Boston Area Community Health Survey. The Journal of urology, 2009;182(2): 616-625.

18. Christ George J., Bushman Wade, Fraser Matthew O. Impact of Diabetes and Obesity on the Prostate and Urethra: Implications to Improved Bladder Dysfunction Understanding and Treatment. Journal of Urology. 2009;182(6S):S38-44. 
19. Al-Shaiji TF, Radomski SB. Relationship between Body Mass Index and Overactive Bladder in Women and Correlations with Urodynamic Evaluation. Int Neurourol J. 2012;16(3):126-31.

20. Cummings JM, Rodning CB. Urinary Stress Incontinence Among Obese Women: Review of Pathophysiology Therapy. Int Urogynecol J. 2000;11(1):41-4.

21. Lee Y-C, Lin G, Wang G, Reed-Maldonado A, Lu Z, Wang L, et al. Impaired contractility of the circular striated urethral sphincter muscle may contribute to stress urinary incontinence in female zucker fatty rats. Neurourol Urodyn. 2017;36(6):1503-10.

22. Wang L, Lin G, Lee $Y-C$, Reed-Maldonado $A B$, Sanford MT, Wang G, et al. Transgenic Animal Model for Studying the Mechanism of Obesity- Associated Stress Urinary Incontinence. BJU Int. 2017;119(2):317-24.

23. Cava E, Yeat NC, Mittendorfer B. Preserving Healthy Muscle during Weight Loss. Adv Nutr.2017;8(3):511-9.

24. Wens I, Dalgas U, Vandenabeele F, Krekels M, Grevendonk L, Eijnde BO. Multiple Sclerosis Affects Skeletal Muscle Characteristics. PLOS ONE. 2014;9(9):e108158.

24. Wingo BC, Young H-J, Motl RW. Body composition differences between adults with multiple sclerosis and BMI-matched controls without MS. Disability and Health Journal. 2018;11(2):243-8. 
10

8

6

4

0
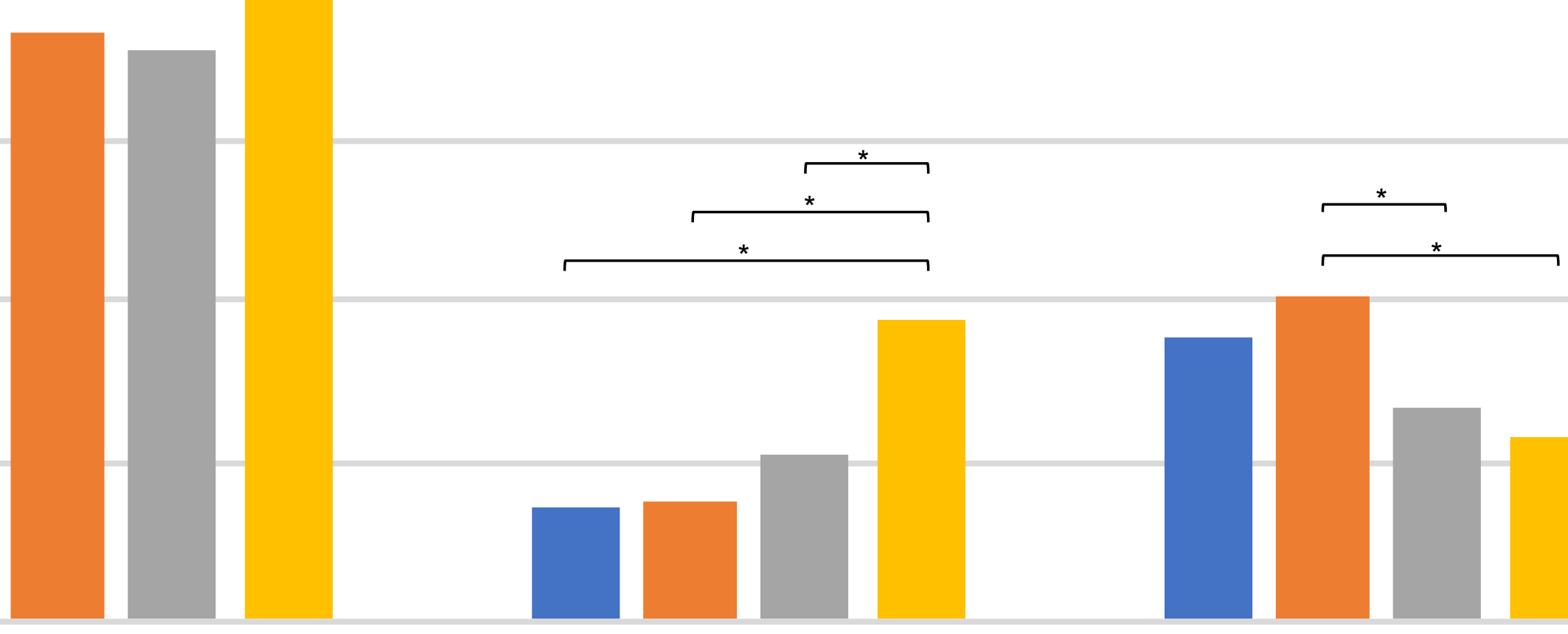

USP HAV

USP IUE

USP dysurie

- Insuffisance pondérale

- Poids normal

Surpoids

Obésité 


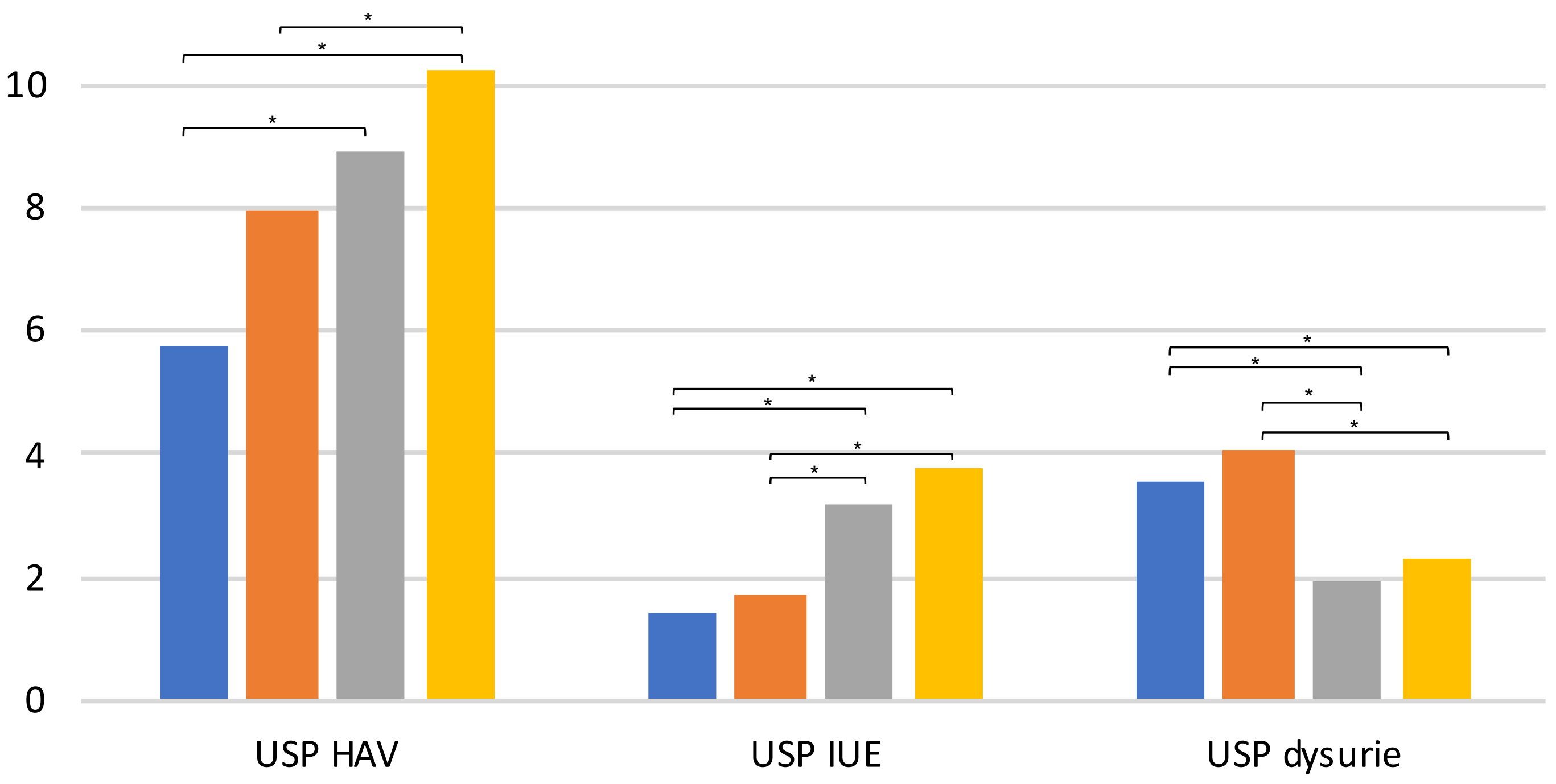

- Insuffisance pondérale $\square$ Poids normal $\square$ Surpoids $\square$ Obésité 